\title{
Production of atmospheric-pressure glow discharge in nitrogen using needle-array electrode
}

\author{
K. Takaki, ${ }^{a)}$ M. Hosokawa, T. Sasaki, S. Mukaigawa, and T. Fujiwara \\ Department of Electrical and Electronic Engineering, Iwate University, 4-3-5 Ueda, Morioka, \\ Iwate 020-8551, Japan
}

(Received 21 December 2004; accepted 3 March 2005; published online 6 April 2005)

\begin{abstract}
An atmospheric pressure glow discharge was generated using a needle-array electrode in nitrogen, and the voltage-current characteristics of the glow discharge were obtained in a range from $1 \mathrm{~mA}$ to $60 \mathrm{~A}$. A pulsed high voltage with short rise time under $10 \mathrm{~ns}$ was employed to generate streamer discharges simultaneously at all needle tips. The large number of streamer discharges prevented the glow-to-arc transition caused by inhomogeneous thermalization. Semiconductor opening switch diodes were employed as an opening switch to shorten the rise time. The glow voltage was almost constant until the discharge current became $0.3 \mathrm{~A}$, whereas the voltage increased with the current higher than $0.3 \mathrm{~A}$. Electron density and temperature in a positive column of the glow discharge at $60 \mathrm{~A}$ were obtained to $1.4 \times 10^{12} \mathrm{~cm}^{-3}$ and $1.3 \mathrm{eV}$ from calculation based on nitrogen swarm data.

(C) 2005 American Institute of Physics. [DOI: 10.1063/1.1905801]
\end{abstract}

Research on atmospheric pressure glow discharges (APGD) is motivated by applications such as instantly activated reflectors and absorbers for electromagnetic radiation, surface modification, thin film deposition, dry etching, remediation and detoxification of gaseous pollution, sterilization, and light sources as gas lasers and excimer radiation. ${ }^{1-4} \mathrm{Gas}$ plasmas can be generated by ionizing a neutral gas. A number of techniques have been developed based on gas ionization. These include: (1) ionization by an external source of radiation such as $x$ rays, electron beams, photoionizing radiation, and (2) through the use of gas discharge. ${ }^{5}$ Gas discharges are by far the most common approach because the efficiency of external ionization is generally rather low and therefore the cost of such a method relatively high. ${ }^{1}$

A gas discharge is created when an electric field of sufficient amplitude is applied to a volume of gas to generate electron-ion pairs by electron impact ionization of the neutral gas. This requires the high density of a power deposition into the weakly ionizing plasma for high pressure gas medium because of short mean free path of electrons. In general, the high density power deposition induces a thermal instability, which causes the change of a discharge mode from a spatially uniform glow to an arc. Much of the efforts in generating stable glow discharges at atmospheric pressure have focused on preventing the onset of instabilities, particularly in the region close to the cathode, because of high electric field and consequently high power deposition compared to that in the positive column of the discharge. ${ }^{6}$

A pulse voltage on preventing the thermal instability is commonly employed in the laser community to generate the glow discharges operated at current densities above threshold for the glow-to-arc transition. The duration of the discharge pulses is chosen to be shorter than the time constant of the dominant instability that causes the transition, typically less than $1 \mu \mathrm{s} .{ }^{7}$ In general, two high voltage pulses from a spiker and a sustainer circuits are combined and apply to the excimer laser gas medium in order to generate uniform glow plasma. This letter describes the generation of APGD in ni-

\footnotetext{
${ }^{a)}$ Electronic mail: takaki@iwate-u.ac.jp
}

trogen gas using pulsed power supply which can generate pulse voltage consisted of a narrow overvoltage pulse and a long width pulse. A needle-array electrode instead of planar electrode was used to generate streamer discharges homogeneously between the electrodes and to keep the glow discharge stable for long duration. The properties of the APGD plasma such as voltage-current $(V-I)$ characteristics, an electron density, and an electron temperature are also described.

A schematic diagram of the experimental facilities is shown in Fig. 1. The grounded electrode plate with rounded edges is made of brass and set in the discharge chamber. The overall diameter and thickness of the electrodes are 10.7 and $1.5 \mathrm{~cm}$, respectively. The needle-array electrode made of brass is set to the upper part of the chamber. The needlearray electrode consists of 331 needles which have a dimension of $1.3 \mathrm{~cm}$ in height and $50 \mu \mathrm{m}$ in radius of the tip. Typical gap length $d$ from needle tip to the electrode surface is $1.0 \mathrm{~cm}$. The discharge chamber is evacuated with a rotary pump and the nitrogen gas is filled into the chamber to atmospheric pressure.

The power supply consists of $8.0 \mathrm{nF}$ capacitor $C$, gap switch, $20 \mu \mathrm{H}$ inductor $L$, and semiconductor opening switch (SOS) diode. The capacitor is charged up negatively to the voltage $V_{0}$ using a dc high voltage power supply. The charges are released from the capacitor by switching-on the gap switch mechanically, as the results, the current flow to the LC circuit through the SOS diode as forward-pumping

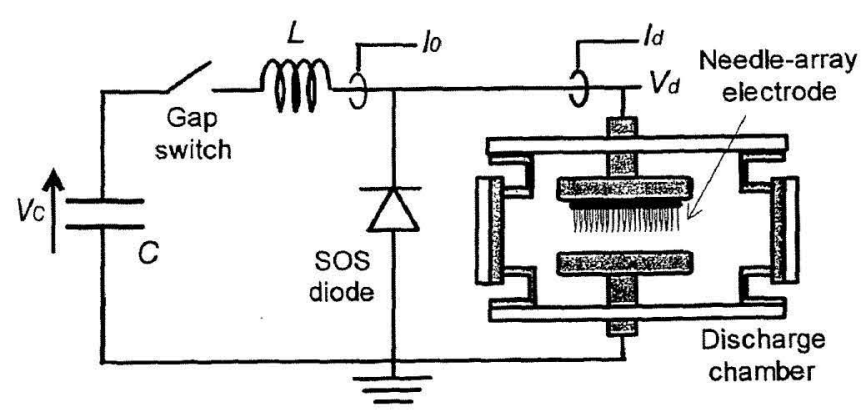

FIG. 1. Schematic diagram of the experimental facilities used for generation of the atmospheric pressure glow discharge. 


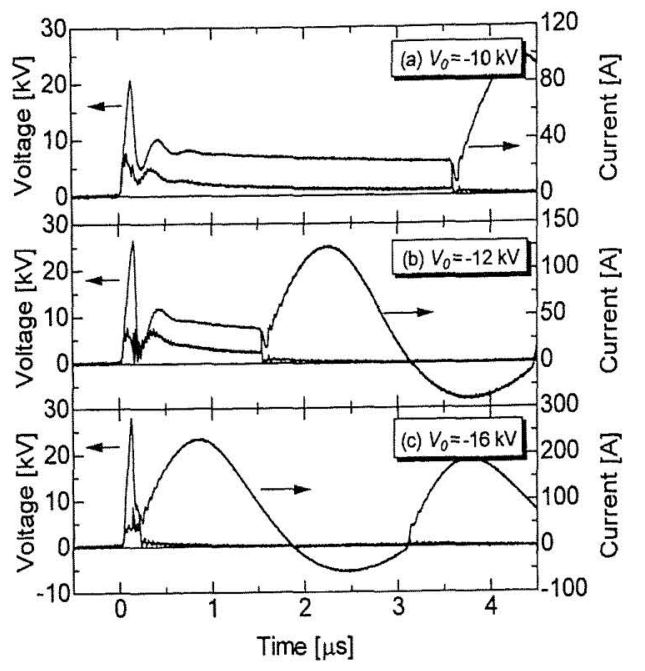

FIG. 2. Voltage and current waveforms of the discharge for various charging voltages.

current. ${ }^{8}$ After the current direction reverses with LC oscillation, the reverse current is injected into the SOS. With the current interrupted by the SOS, the high voltage pulse is applied to the needle-array electrode as a short nanosecond pulse. The current and voltage are measured with Pearson 2878 current transformers and Sony-Tektronix high-voltage $\mathrm{P} 6015 \mathrm{~A}$ probes. The signals stored in a digitizing oscilloscope Tektronix TDS3054B are transmitted to a personal computer through a GP-IB cable in order to calculate the energy consumed in the discharge.

Representative waveforms of the transient glow discharge current and the voltage between the electrodes for three different charging voltages $V_{0}$ are shown in Fig. 2 . When the narrow overvoltage pulse is applied to the needlearray electrode, the breakdown occurs and the discharge current rises rapidly. The long pulse voltage, which has an altitude of the voltage lower than charging voltage, follows the overvoltage pulse and keeps the glow discharge in a quasistable state, i.e., a transient glow phase. The glow discharge is sustained for $3 \mu \mathrm{s}$ at $-10 \mathrm{kV}$ charging voltage as shown in Fig. 2(a) with glow current from 10 to $3 \mathrm{~A}$. At the time of $3.5 \mu$ s, the voltage collapses to a few hundred volts with glow-to-arc transition characterized by an appearance of a luminous spot on the cathode surface. ${ }^{6}$ Figure 2 clearly shows that the quasistable state glow discharge current increases with increasing the charging voltage whereas the duration of the glow phase decreases.

Chalmers suggested that the criterion for a glow-to-arc transition is that a certain quantity of energy is consumed in the gap during the glow phase. ${ }^{9}$ Figure 3 shows the plots

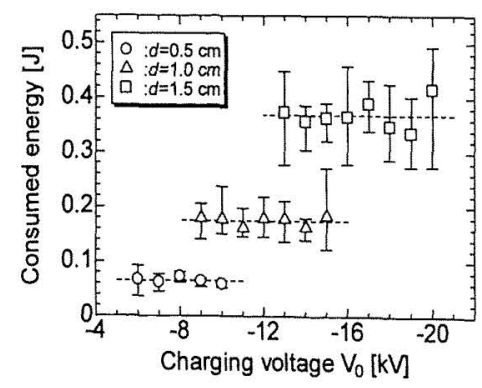

FIG. 3. Consumed energy for glow-to-arc transition as a function of charging voltage at various gap lengths.

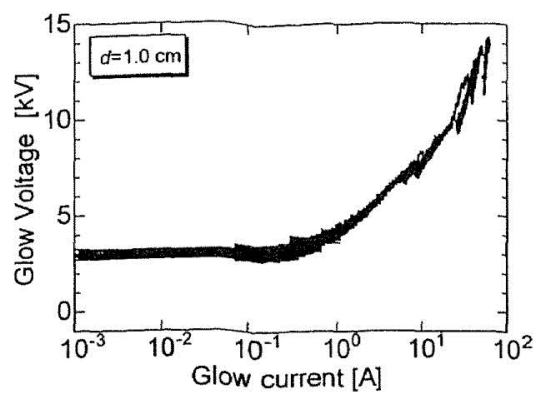

FIG. 4. Current-voltage characteristics of nitrogen glow discharge at $1.0 \mathrm{~cm}$ gap length.

of the energy consumed in the discharge during the glow phase as a function of charging voltage for three different gap lengths. The consumed energies are calculated with time integration of the power, which is obtained by multiplying the discharge current with the voltage, during the time duration of the glow phase. The consumed energies are almost independent on the charging voltage though the measured values are scattered. The mean value of the consumed energy increases with increasing the gap length, 0.07, 0.17, and $0.37 \mathrm{~J}$ for $0.5,1.0$, and $1.5 \mathrm{~cm}$ in gap length, respectively. These values of the consumed energy can be used as a criterion for a glow-to-arc transition. ${ }^{6,9}$ The energy input to the discharge can be changed with the capacitor $C$, the inductor $L$, and/or inserting a damping resistor into the circuit in series.

A lot of processes in a direct current glow plasma are governed by the $V-I$ characteristics of the discharge. ${ }^{10}$ The $V-I$ characteristics of a transient glow discharge can be obtained in wide current range from milliamps to larger than 100 A using the discharge current and the voltage waveforms. " Figure 4 shows the $V-I$ characteristics of the quasistable glow discharge in atmospheric pressure nitrogen gas at $1.0 \mathrm{~cm}$ gap length obtained in the same manner. The lines were overwritten at different charging voltage in order to obtain in wide current range. The glow voltage is almost constant, $3 \mathrm{kV}$, when the glow current is lower than $0.3 \mathrm{~A}$. This independency of the voltage on the current is a typical feature of a normal glow discharge. "The glow voltage increases from 3 to $14 \mathrm{kV}$ with increasing glow current from 0.3 to $60 \mathrm{~A}$. The glow voltage is almost constant at lower current than $0.3 \mathrm{~A}$ while it increases with increasing current in a range larger than $0.3 \mathrm{~A}$. This fact implies that the discharge changes from a normal glow to an abnormal glow at the discharge current of $0.3 \mathrm{~A}$.

The value of electric field in the positive column of the glow discharge can be predicted from glow voltage and cathode fall voltage, which is determined by "zero length voltage" extrapolating the potential distribution across glow discharge to zero gap length. ${ }^{12}$ In the normal glow mode $(<0.3 \mathrm{~A})$, the electric field of the positive column is roughly obtained to be $2.8 \mathrm{kV} / \mathrm{cm}$, which is consistent to $1.1 \times 10^{-16} \mathrm{~V} \mathrm{~cm}^{2}$ in reduced electric field $E / N$. The electron temperature $T_{e}$ can be calculated from swarm parameters of electrons in nitrogen as well-known Einstein's equation, $k_{B} T_{e} / e \approx D_{e} / \mu_{e}$, where $\mu_{e}, k_{B}$, and $D_{e}$ are drift mobility, Boltzmann constant $\left(1.38 \times 10^{-23} \mathrm{~J} \mathrm{~K}^{-1}\right)$ and the diffusion constant, which can be expressed as a function of $E / N{ }^{13}$ As the results, the electron temperature is predicted to be $0.9 \mathrm{eV}$ for the normal glow and $1.3 \mathrm{eV}$ for abnormal glow at $60 \mathrm{~A}$ in glow current. The averaged electron density can be 
estimated using formula, $N_{e}=j / e W$, where $j, e$, and $W$ are current density, electron charge, and electron drift velocity, which also can be calculated as a function of $E / N .^{13}$ The current density $j$ in the abnormal glow mode is determined by division of the total glow current by the cross section of the positive column; we assumed the cross section to $42.7 \mathrm{~cm}^{2}$, which was obtained by the overall diameter of the needle array. As the results, the electron densities are estimated to be $0.8 \times 10^{10} \mathrm{~cm}^{-3}$ for normal glow $(<0.3 \mathrm{~A})$ and to be $1.4 \times 10^{12} \mathrm{~cm}^{-3}$ for abnormal glow at $60 \mathrm{~A}$ in glow current.

The authors would like to thank Professor H. Akiyama of Kumamoto University, H. Mase of Ibaraki University, and N. Sato of Tohoku University for their valuable discussions and comments. The authors would like to thank Mr. S. Kato of Iwate University for his cooperation. This work was supported by a Grant-In-Aid of Science Research from Japan
Ministry of Education, Science and Culture (JSPS Fellowship No. 15075101).

'R. J. Stark and K. H. Schoenbach, J. Appl. Phys. 85, 2075 (1999).

${ }^{2}$ R. Prat, Y. J. Koh, Y. Babukutty, M. Kogoma, S. Okazaki, and M. Kodama, Polymer 41, 7355 (2000).

${ }^{3}$ T. C. Montie, K. Kelly-Wintenberg, and J. R. Roth, IEEE Trans. Plasma Sci. 28, 41 (2000).

${ }^{4}$ M. J. Shenton and G. C. Stevens, J. Phys. D 34, 2761 (2001).

${ }^{5}$ E. E. Kunhardt, IEEE Trans. Plasma Sci. 28, 189 (2000).

${ }^{6}$ K. Takaki, D. Kitamura, and T. Fujiwara, J. Phys. D 33, 1369 (2000).

${ }^{7}$ Yu. D. Korolev and G. A. Mesyats, Physics of Pulsed Breakdown in Gases (URO, Yekaterinburg, 1998), Chap. 6.

${ }^{8}$ M. I. Yalandin, S. K. Lyubutin, M. R. Oulmascoulov, S. N. Rukin, V. G. Shpak, S. A. Shunailov, and B. G. Slovikovsky, IEEE Trans. Plasma Sci. 30, 1700 (2002).

${ }^{9}$ I. Chalmers, J. Phys. D 4, 1147 (1971).

${ }^{10}$ L. J. Denes and J. J. Lowke, Appl. Phys. Lett. 23, 130 (1973).

${ }^{11}$ K. Takaki, D. Taguchi, and T. Fujiwara, J. Appl. Phys. 78, 2646 (2001).

${ }^{12} \mathrm{M}$. Cavenor and J. Meyer, Aust. J. Phys. 22, 155 (1969).

${ }^{13}$ Y. Nakamura, J. Phys. D 20, 933 (1987). 